Agronomía Costarricense 35(2): 33-47. ISSN:0377-9424 / 2011

www.mag.go.cr/rev_agr/index.html www.cia.ucr.ac.cr

\title{
CARACTERIZACIÓN DEL DESARROLLO Y ABSORCIÓN DE NUTRIMENTOS DEL HÍBRIDO DE MAÍZ HC-57
}

\author{
Róger Fallas $^{1 / *}$, Floria Bertsch $^{*}$, Carlos Echandi ${ }^{* *}$, Carlos Henríquez
}

\begin{abstract}
Palabras clave: Zea mays, curvas de absorción, macronutrimentos, micronutrimentos, fenología, grados día.
\end{abstract}
Keywords: Zea mays, nutrient absorption curves, macronutrients, micronutrients, phenology, heat units.

Recibido: 21/09/10

\section{RESUMEN}

En la Estación Experimental Agrícola Fabio Baudrit Moreno, Alajuela, Costa Rica se realizó un ensayo con el objetivo de determinar la absorción de nutrimentos por parte del híbrido comercial de maíz HC-57. Se sembraron un total de 120 plantas, a las que se les dio buen manejo hídrico y nutricional. Se realizaron muestreos aproximadamente cada 15 días y se colectaron un total de 9 plantas por época de muestreo. A dichas plantas se les estimó la altura, se les cuantificó el número de hojas y fueron divididas en secciones (raíz, follaje y mazorca). Se secaron en estufa a $80^{\circ} \mathrm{C}$ y se estimó el peso seco. Posteriormente se determinó la concentración de nutrimentos en el Laboratorio de Suelos y Foliares del Centro de Investigaciones Agronómicas, Universidad de Costa Rica y se obtuvieron las curvas de absorción de nutrimentos para macro y microelementos. Para un rendimiento de 50000 mazorcas.ha ${ }^{-1}$, con un peso seco de 141,5 g por mazorca, se presentó una absorción máxima total por hectárea de $111 \mathrm{~kg}$ de $\mathrm{N}, 43 \mathrm{~kg}$ de $\mathrm{P}_{2} \mathrm{O}_{5}, 168$ $\mathrm{kg}$ de $\mathrm{K}_{2} \mathrm{O}, 26 \mathrm{~kg}$ de $\mathrm{CaO}, 28 \mathrm{~kg}$ de $\mathrm{MgO}, 10 \mathrm{~kg}$ de $\mathrm{S}, 3200 \mathrm{~g}$ de Fe, $100 \mathrm{~g}$ de $\mathrm{Cu}, 400 \mathrm{~g}$ de $\mathrm{Zn}, 300$ $\mathrm{g}$ de Mn y $100 \mathrm{~g}$ de B. Además, se realizó una caracterización del ciclo de desarrollo del híbrido, relacionada con las condiciones climáticas

1 Autor para correspondencia. Correo electrónico: rafallasc@gmail.com

Centro de Investigaciones Agronómicas, Universidad de Costa Rica, San José, Costa Rica.
Aceptado: 26/07/11

\section{Nutrient absorption and phenology development of the Costa Rican corn hybrid} HC-57. The aim of the study was to investigate the total nutrient absorption in the corn hybrid HC-57. The experiment was carried out at the Agricultural Experimental Station Fabio Baudrit Moreno of the University of Costa Rica, in Alajuela Costa Rica. A plot with 120 plants under optimal growth conditions was used. Plants were sampled every 15 days using 9 units per sampling. The entire plant was collected and the height and number of leaves determined. Then the plants were divided into roots, foliage and cobs, dried at $80^{\circ} \mathrm{C}$, weighed and analyzed at the Laboratory of the Agronomic Research Center of the University of Costa Rica. For macro and micronutrients, uptake curves were obtained. For a yield estimate of 50000 cobs.ha ${ }^{-1}$, with a dry weight of $141,5 \mathrm{~g}$ per cob, the maximum nutrient absorption per hectare was $111 \mathrm{~kg}$ of $\mathrm{N}, 43 \mathrm{~kg}$ $\mathrm{P}_{2} \mathrm{O}_{5}, 168 \mathrm{~kg} \mathrm{~K}{ }_{2} \mathrm{O}, 26 \mathrm{~kg} \mathrm{CaO}, 28 \mathrm{~kg} \mathrm{MgO}, 10 \mathrm{~kg}$ S, $3200 \mathrm{~g} \mathrm{Fe}, 100 \mathrm{~g} \mathrm{Cu}, 400 \mathrm{~g} \mathrm{Zn}, 300 \mathrm{~g} \mathrm{Mn}$ and $100 \mathrm{~g} \mathrm{~B}$. It was found that the Corn hybrid HC-57 required approximately 1500 growing degree days to complete its development cycle and 1254 growing degree days to reach the R3 phenological stage, at which it is harvested as sweet corn. The

\footnotetext{
** Estación Experimental Fabio Baudrit Moreno, Universidad de Costa Rica, Alajuela, Costa Rica.
} 
que permitió asociar la información de absorción con los grados día temperatura, estimados por el método residual modificado descrito por Yzarra et al. (2009), con el propósito de generar una predicción del comportamiento del ciclo y la absorción de nutrimentos de este híbrido en otras localidades. El híbrido HC-57 requirió un aproximado de 1500 grados día temperatura para completar su ciclo de desarrollo y 1254 grados día temperatura para alcanzar la etapa fenológica $\mathrm{R} 3$, en la cual se realiza la cosecha para elote.

\section{INTRODUCCIÓN}

El maíz es uno de los cultivos de mayor importancia en el mundo que junto con el arroz y el trigo se posiciona como uno de los principales cereales (APS 1984). En Costa Rica el híbrido de maíz HC-57 es un material que por sus características de adaptación a diferentes climas, tolerancia a pudrición de mazorca, buen cuaje, sabor y textura, se ha posicionado como una muy buena opción productiva a nivel nacional (Girasol 2006).

Aunque el híbrido HC-57 tiene varios años en el mercado, no se ha caracterizado su ciclo fenológico, ni se tiene cuantificada su absorción de nutrimentos.

Pese a que las etapas fenológicas del cultivo son similares entre variedades e híbridos de maíz, la duración entre cada una de ellas puede variar por diferentes factores como son las características genéticas propias, las fechas de siembra, las localidades y las condiciones climáticas (Iowa State University 1992).

También se ha encontrado que la tasa de desarrollo de los cultivos está relacionada con la temperatura (Iowa State University 1992, Miller y Brandt 2001), lo que ha promovido la definición de otro concepto en el desarrollo de los cultivos, llamado "tiempo térmico", el cual sustituye al tiempo cronológico (Misle 2003).

La utilización del concepto de "tiempo térmico" o "grados día temperatura" en el cultivo information on nutrient absorption and on the respective growing degree days is presented for all the stages evaluated, in order to create a link between both parts.

del maíz, así como en otros cultivos como rosa, frijol y melón, ha demostrado una amplia validez en la predicción de eventos o estados fenológicos; hay estudios que se basan en la acumulación de calor como unidad de medición y no sobre unidades diarias, como generalmente se realiza (Misle 2003, Rodríguez y Flórez 2006, Barrios y López 2009). El asocio del tiempo térmico a la absorción de nutrimentos por los cultivos también ha sido documentado por otros autores (Misle 2003).

Por otra parte, es importante conocer el desarrollo del cultivo y las etapas de mayor necesidad de nutrimentos durante la producción pues de esa forma se puede ofrecer al cultivo las condiciones adecuadas para obtener rendimientos óptimos. En el caso del maíz, García (2005) menciona que aportar condiciones óptimas en la etapa de floración es imprescindible para lograr buenos rendimientos.

El objetivo de este trabajo fue cuantificar y caracterizar la absorción de nutrimentos por parte del híbrido HC-57, y relacionar esta absorción con el desarrollo fenológico y la acumulación de grados día temperatura.

\section{MATERIALES Y MÉTODOS}

El experimento se llevó a cabo en la Estación Experimental Agrícola Fabio Baudrit Moreno (EEFBM), localizada en Barrio San José de Alajuela, a $840 \mathrm{msnm}$, la cual clasifica según 
Holdridge (1982) como una zona de vida de bosque húmedo premontano, con un clima subhúmedo caliente y estación seca bien definida. La precipitación pluvial promedio anual es de 1940 mm distribuidos de mayo a diciembre, con humedad relativa diaria de $78 \%$, evaporación anual de $1876 \mathrm{~mm}$, radiación solar total anual 8428,2 MJ y brillo solar 2396,5 h.

Se sembraron 120 plantas de maíz del híbrido HC-57, siguiendo el patrón convencional utilizado en la EEFBM, que consiste en una densidad de 50000 plantas.ha- $^{-1}$, espaciadas a $0,8 \mathrm{~m}$ entre hileras y $0,5 \mathrm{~m}$ entre planta, con 2 plantas en cada punto de siembra. Las plantas recibieron el manejo hídrico y nutricional que normalmente se le aplica a los experimentos con ese híbrido de maíz dentro de la estación. La fertilización utilizada fue de $150 \mathrm{~kg} \cdot \mathrm{ha}^{-1}$ de N, 22,5 kg.ha ${ }^{-1}$ de $\mathrm{P}_{2} \mathrm{O}_{5}$ y $10 \mathrm{~kg}$ ha ${ }^{-1}$ de $\mathrm{K}_{2} \mathrm{O}$, acorde a las condiciones generales del suelo. El estado de la plantación fue adecuado a lo largo de todo el ciclo de modo que se puede asumir que los resultados responden a una plantación regular del híbrido HC-57 bajo esas condiciones.

Se realizaron evaluaciones en 7 diferentes épocas del ciclo de desarrollo del cultivo, con inicio a los 22 días después de la siembra (dds). En cada una de las evaluaciones se determinó la etapa fenológica en la que se encontraba la plantación, para lo cual se utilizó el patrón establecido en el estado de Iowa, Estados Unidos (Iowa State University 1992).

La muestra recolectada fue de 9 plantas en cada época de muestreo, a las cuales se les estimó de forma individual la altura desde la base hasta el "collarín" o lígula superior visible. Posteriormente, cada planta se dividió en raíz, follaje y mazorca, tejidos que fueron secados en estufa a $80^{\circ} \mathrm{C}$ por 3 días para determinar el peso seco de cada parte.

Para estimar la concentración de nutrimentos presentes en los tejidos, las plantas de las 3 repeticiones se unieron, y se enviaron al Laboratorio de Suelos y Foliares del CIA-UCR. Con la información obtenida y con el respectivo asocio entre la concentración y el peso seco de cada tejido, se obtuvo el consumo efectuado por cada uno de ellos en cada etapa y así desarrollar las curvas de absorción de nutrimentos del cultivo.

También se relacionó la información climatológica de temperatura, proveniente de la estación Clase B ubicada en la propia EEFBM con las curvas de absorción de nutrimentos. Para el cálculo de los grados día temperatura (GDT) se utilizó la fórmula de Ometto, que se describe en Yzarra et al. 2009 y ha sido utilizada a lo largo del tiempo por varios autores (Avilán y Rodríguez 1995, Hamada y Silveira Pinto 2001,Yzarra et al. 2009); el cálculo también se detalla a continuación:

$$
\begin{aligned}
& \text { Cuando } T m>T b \text { y } T M<T B: \\
& \text { GDT }=\left(\frac{(T M-T m)}{2}\right)+(T m-T b)
\end{aligned}
$$

Cuando $T m>T b$ y $T M>T B$ :

$$
G D T=\left(\frac{2(T M-T m)(T m-T b)+(T M-T m)^{2}-(T M-T B)^{2}}{2(T M-T m)}\right)
$$

Donde:

TM=Temperatura máxima diaria $\mathrm{Tm}=$ Temperatura mínima diaria $\mathrm{TB}=$ Temperatura base máxima $\mathrm{Tb}=$ Temperatura base mínima

Se usó una temperatura base mínima de $10^{\circ} \mathrm{C}$ y una máxima de $30^{\circ} \mathrm{C}$ en concordancia con las recomendaciones establecidas en la literatura (Wiebold, sa).

La obtención de los GDT en cada etapa de muestreo, ofrece una herramienta adicional al productor para transferir la información generada en la EEFBM a las diferentes localidades del país donde se cultiva este híbrido.

\section{RESULTADOS Y DISCUSIÓN}

\section{Desarrollo de la planta y estado fenológico}

En la Figura 1 se presenta el ciclo de desarrollo del híbrido HC-57 expresado en términos de número de hojas y altura.

El híbrido de maíz HC-57 presenta una etapa de crecimiento vegetativo que se extiende 


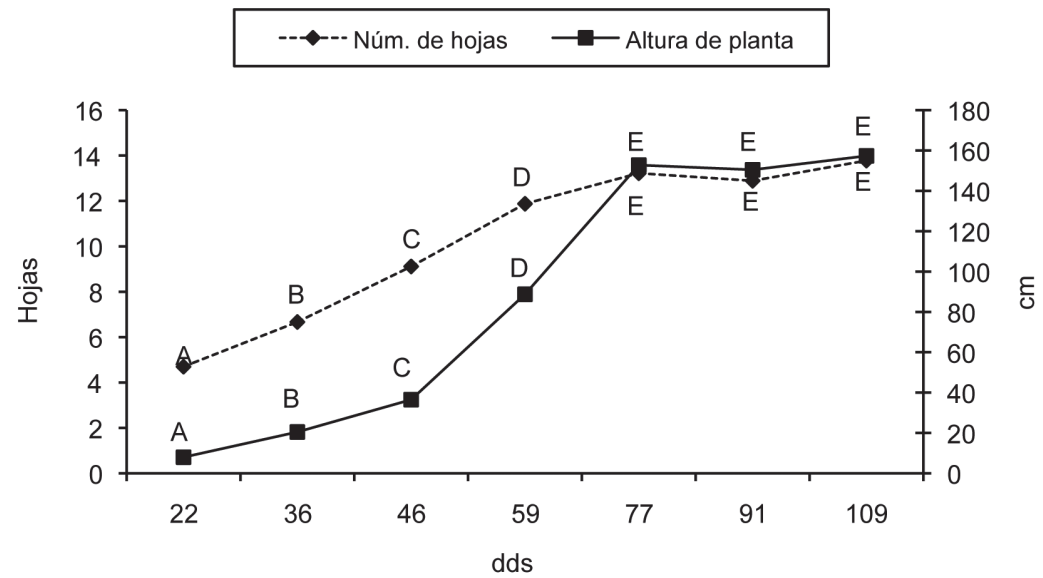

Fig.1. Altura y número de hojas del híbrido de maíz HC-57 durante su ciclo de desarrollo en la Estación Experimental Fabio Baudrit Moreno. Alajuela, Costa Rica. Año. 2009.

desde la etapa inicial, hasta antes de los $77 \mathrm{dds}$ (de V1 a VT) en la cual incrementa significativamente su número de hojas y su altura; después de esta etapa la planta no presenta ningún incremento significativo en ese último aspecto (Figura 1). Esta misma situación acontece con la variable peso seco del follaje (tallo y hojas) (Figura 2).
La estabilización en la producción de material vegetativo observada a partir de los 77 dds en la emisión de hojas nuevas, altura de planta y peso seco del follaje, corresponde con el inicio del proceso reproductivo de la planta, e inicio de formación de la mazorca, la cual se caracteriza por un aumento significativo de su peso desde la

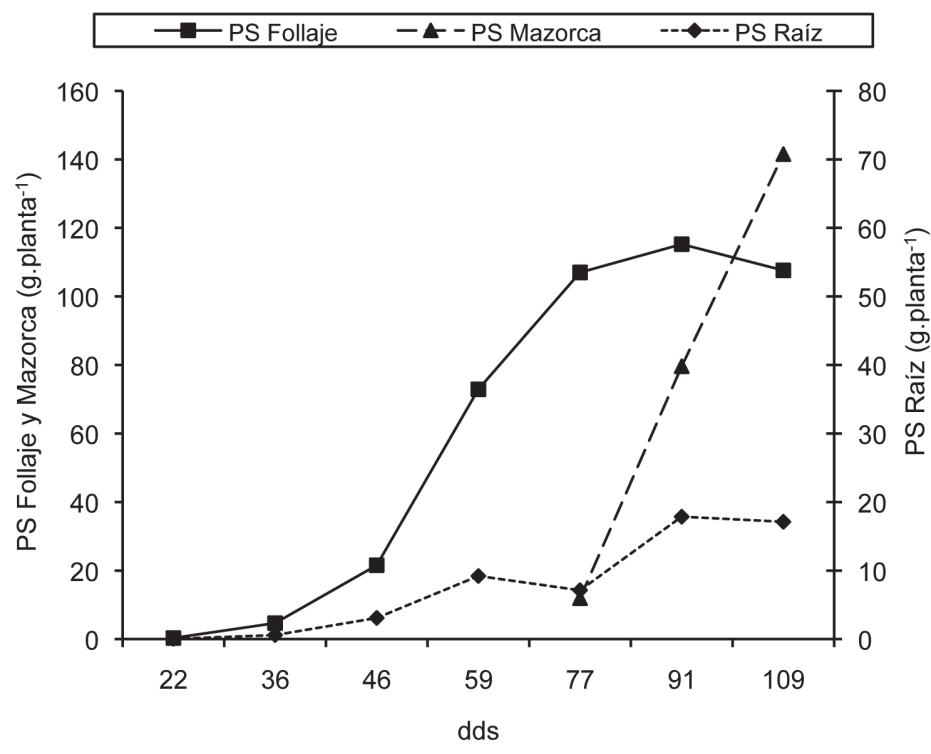

Fig. 2. Peso seco de raíz, follaje y mazorca del híbrido de maíz HC-57 (g.planta-1) durante su ciclo de desarrollo en la estación Experimental Fabio Baudrit Moreno, Alajuela, Costa Rica. Año 2009. 
etapa R1 hasta el final del ciclo de desarrollo del cultivo (Madurez fisiológica) (Figura 2).

Con las mediciones efectuadas no fue posible determinar el momento en que ocurre el proceso de diferenciación del meristemo superior (flor masculina) ya que el meristemo al estar recubierto por las hojas nuevas, obstaculizaron su visualización. Por esta razón, las variables altura de planta y emisión de hojas nuevas no son de utilidad para determinar dicho evento fenológico, y lo conlleva a ser detectado sólo mediante muestreos destructivos.

En relación con la cuantificación del peso seco de la raíz se considera que, en proporción al trabajo que representa, es una variable que aporta poca información al estudio, ya que entre muchas de las etapas de desarrollo no se presentaron diferencias significativas. Esta situación unida al hecho que el procedimiento de medición de raíz es muy laborioso, tanto durante el proceso de extracción como durante la remoción del suelo adherida a las mismas a la hora del lavado, permite recomendar para futuros trabajos de absorción, la posibilidad de prescindir de este tejido. En el caso concreto del híbrido de maíz HC-57 el máximo porcentaje de raíz obtenido en relación con el peso total de la planta correspondió con menos de un $7 \%$.

Como se puede observar en el Cuadro 1, el ciclo de desarrollo del HC-57 duró 109 días para alcanzar la etapa de madurez fisiológica y 91 días para ser cosechado para elote, aspecto esperable bajo las condiciones de producción del lugar. No obstante debe considerarse que dicha información puede variar en diferentes ciclos de siembra y en las diferentes localidades del país donde es cultivado el híbrido, ya que condiciones ambientales como temperatura tienen un efecto directo sobre los procesos fisiológicos del desarrollo de las plantas (Casasola y Tiessen 2009).

Además se ha determinado que el híbrido presenta un desarrollo dependiente del fotoperiodo, obteniéndose una mayor altura de planta en fotoperiodos crecientes y menor en decrecientes (Echandi 2010). Comunicación personal. Estación Experimental Fabio Baudrit Moreno. Alajuela, Costa Rica, cabe destacar que la siembra de este ensayo se realizó a finales de setiembre, donde el fotoperiodo es decreciente, por lo que puede existir cierta variación de los datos con otras épocas de siembra.

Como se mencionó anteriormente el uso de los GDT tiene gran utilidad en la predicción

Cuadro 1. Ciclo fenológico del híbrido de maíz HC-57 y grados acumulados de temperatura obtenidos en la Estación Experimental Fabio Baudrit Moreno, Alajuela, Costa Rica.

\begin{tabular}{cccc}
\hline dds & Acúmulo GDT & Estado fenológico & Observaciones \\
\hline 22 & 318 & V5 & \\
36 & 501 & V7 & Meristermo superior diferenciado \\
46 & 632 & V9 & \\
59 & 811 & V12-VT & \\
77 & 1062 & R1-R2 & Cosecha para elote: grano lechoso \\
91 & 1254 & R3 & Cosecha para grano \\
\hline
\end{tabular}

dds=días después de la siembra.

GDT=Grados día temperatura calculado mediante la fórmula de Ometo (Yzarra et al. 2009), con una temperatura base mínima de $10^{\circ} \mathrm{C}$ y máxima de $30^{\circ} \mathrm{C}$. 
de las etapas fenológicas por las que atraviesa el maíz y en la predicción de la época de cosecha. El híbrido HC-57 requirió un aproximado de 1500 GDT para completar su ciclo de desarrollo y se requirieron un total de 1254 para alcanzar la etapa fenológica R3, en la cual se realiza la cosecha para elote (Cuadro 1).

\section{Concentración de nutrimentos según tejido}

El promedio de las concentraciones de nutrimentos en cada uno de los tejidos a lo largo del ciclo del maíz HC-57 y la diferencia estadística entre medias se presentan en el Cuadro 2.

Follaje. Según se observa en el Cuadro 2, la concentración de N, P y K en el follaje decrece en forma significativa conforme avanzan las etapas, pues pasa de valores cercanos a 4\%, 0,3\% y $4,7 \%$ respectivamente, a concentraciones 6 y 3 veces menores, $0,67 \%, 0,05 \%$ y $1,49 \%$, respectivamente, al final del ciclo.

En el caso del Ca y $\mathrm{Mg}$ no se encontraron diferencias en la concentración para la mayoría de las etapas evaluadas, salvo en la etapa más joven donde en general, todos los nutrimentos se encuentran más concentrados. Estas diferencias no fueron significativas estadísticamente, ni mayores a las variaciones aceptadas como máximas para los análisis foliares de laboratorio (Corrales et al. 2005).

En relación con los elementos menores la tendencia es más errática, aunque en general,

Cuadro 2. Concentraciones promedio de nutrimentos en los diferentes tejidos del híbrido de maíz HC-57 en la Estación Experimental Fabio Baudrit Moreno, Alajuela, Costa Rica.

\begin{tabular}{|c|c|c|c|c|c|c|c|c|c|c|c|c|}
\hline \multirow[b]{2}{*}{ Tejido } & \multirow[b]{2}{*}{ dds } & \multicolumn{6}{|c|}{$\%$} & \multicolumn{5}{|c|}{$\mathrm{mg} \cdot \mathrm{kg}^{-1}$} \\
\hline & & $\mathrm{N}$ & $\mathrm{P}$ & $\mathrm{K}$ & $\mathrm{Ca}$ & $\mathrm{Mg}$ & $\mathrm{S}$ & $\mathrm{Fe}$ & $\mathrm{Cu}$ & $\mathrm{Zn}$ & $\mathrm{Mn}$ & B \\
\hline \multirow{3}{*}{ Mazorca } & 77 & $1,92 \mathrm{~B}$ & $0,31 \mathrm{~B}$ & $1,73 \mathrm{C}$ & 0,07 & 0,14 & $0,13 \mathrm{~B}$ & $156 \mathrm{~B}$ & $5 B$ & $29 \mathrm{~A}$ & $18 \mathrm{C}$ & $9 B$ \\
\hline & 91 & $1,05 \mathrm{~A}$ & $0,22 \mathrm{~A}$ & $1,13 \mathrm{~B}$ & 0,05 & 0,13 & $0,09 \mathrm{~A}$ & $84 \mathrm{~A}$ & $2 \mathrm{~A}$ & $27 \mathrm{~A}$ & $14 \mathrm{~B}$ & $6 \mathrm{~A}$ \\
\hline & 109 & $0,96 \mathrm{~A}$ & $0,22 \mathrm{~A}$ & $0,58 \mathrm{~A}$ & 0,03 & 0,13 & $0,08 \mathrm{~A}$ & $65 \mathrm{~A}$ & $2 \mathrm{~A}$ & $25 \mathrm{~A}$ & $8 B$ & $5 \mathrm{~A}$ \\
\hline \multirow{7}{*}{ Follaje } & 22 & $3,98 \mathrm{~F}$ & $0,3 \mathrm{D}$ & $4,7 \mathrm{E}$ & 0,52 & 0,37 & 0,27 & 2221 & 16 & 31 & 58 & 10 \\
\hline & 36 & $3,35 \mathrm{~F}$ & $0,2 \mathrm{C}$ & $3,6 \mathrm{D}$ & 0,21 & 0,14 & 0,16 & 165 & 6 & 15 & 26 & 5 \\
\hline & 46 & $2,74 \mathrm{D}$ & $0,2 \mathrm{C}$ & $2,9 \mathrm{C}$ & 0,22 & 0,14 & 0,15 & 239 & 6 & 15 & 28 & 7 \\
\hline & 59 & $2,27 \mathrm{C}$ & $0,2 \mathrm{C}$ & $2,6 \mathrm{~B}$ & 0,23 & 0,15 & 0,14 & 144 & 6 & 19 & 35 & 9 \\
\hline & 77 & $1,22 \mathrm{~B}$ & $0,14 \mathrm{~B}$ & $1,6 \mathrm{~A}$ & 0,23 & 0,14 & 0,10 & 183 & 5 & 17 & 37 & 12 \\
\hline & 91 & $1,08 \mathrm{AB}$ & $0,11 \mathrm{~B}$ & $1,4 \mathrm{~A}$ & 0,27 & 0,15 & 0,09 & 123 & 4 & 37 & 38 & 13 \\
\hline & 109 & $0,67 \mathrm{~A}$ & $0,05 \mathrm{~A}$ & $1,5 \mathrm{~A}$ & 0,25 & 0,14 & 0,07 & 88 & 4 & 15 & 25 & 11 \\
\hline \multirow{7}{*}{ Raíz } & 22 & 2,00 & 0,19 & 1,86 & 0,31 & 0,15 & 0,20 & 13515 & 70 & 39 & 116 & 35 \\
\hline & 36 & 2,10 & 0,13 & 1,75 & 0,16 & 0,10 & 0,15 & 4982 & 36 & 17 & 43 & 14 \\
\hline & 46 & 1,74 & 0,13 & 1,94 & 0,13 & 0,08 & 0,14 & 3411 & 39 & 16 & 93 & 12 \\
\hline & 59 & 1,57 & 0,10 & 1,82 & 0,09 & 0,06 & 0,12 & 2906 & 26 & 13 & 26 & 10 \\
\hline & 77 & 0,88 & 0,07 & 1,66 & 0,08 & 0,05 & 0,09 & 1389 & 21 & 11 & 19 & 5 \\
\hline & 91 & 0,79 & 0,07 & 1,56 & 0,11 & 0,05 & 0,10 & 2398 & 34 & 34 & 27 & 8 \\
\hline & 109 & 0,69 & 0,06 & 1,66 & 0,09 & 0,05 & 0,07 & 1562 & 18 & 13 & 17 & 6 \\
\hline
\end{tabular}

Letras distintas indican diferencias significativas $(\mathrm{p}<=0,05)$. 
tienden a decrecer. Sólo en el caso de B, la concentración en las etapas iniciales es muy similar a la de las etapas finales.

Mazorca. En el caso de la mazorca se evaluó la concentración en 3 etapas fenológicas. Cuando hubo diferencias, éstas se presentaron entre las 2 primeras etapas R(1-2) y R3 (Cuadro 2). La única disminución que resulta relevante de destacar es la de $\mathrm{K}$, ya que de concentraciones de $1,73 \%$ semejantes a las del follaje a los 77 dds, conforme se consolidaba la mazorca, bajaron a los 109 dds a la tercera parte $(0,58 \%)$.

Raíz. Sobre las concentraciones en la raíz, como ocurre con la mayoría de los cultivos, la variación entre los datos es la más alta y resulta difícil establecer tendencias claras. No obstante, en general, las concentraciones fueron en descenso con la edad.

\section{Curvas de absorción de nutrimentos}

Como se mencionó anteriormente, las curvas de absorción de nutrimentos para el híbrido HC-57 se elaboraron al relacionar el peso seco de la muestra (Figura 2), con la concentración respectiva de cada tejido en las diferentes etapas de crecimiento (Cuadro 2). En las Figuras 3 y 4 se resumen estas curvas de absorción para los nutrimentos mayores y menores, respectivamente.

\section{Macronutrimentos}

Se encontró que la absorción de $\mathrm{N}$ por el híbrido HC-57, está definida según las etapas fenológicas, es decir hasta antes de los 59 dds (VT) la planta absorbe la mayor cantidad del elemento; el $80 \%$ del total requerido en su ciclo de desarrollo completo corresponde a lo absorbido en esta etapa y es consumida principalmente por el follaje.

Después de los 59 dds las necesidades de $\mathrm{N}$ por la planta son menores, y más bien se muestra una reducción en el contenido de este elemento en el follaje y en la raíz. Este comportamiento obedece a la formación de estructuras reproductivas, ya que a partir de ese momento la planta se encuentra en el estado fenológico VT, que significa que inicia la formación de la mazorca (Cuadro 1). La reducción en el contenido de nutrimentos en el follaje y la raíz podría obedecer a un efecto de "exportación" o translocación de nutrimentos hacia las estructuras reproductivas (Bertsch 2003), donde el follaje se presenta como el tejido que aporta la mayor cantidad de nutrimentos hacia la formación de la mazorca.

En el caso del $\mathrm{P}$, el comportamiento a nivel del follaje presenta un patrón similar al del $\mathrm{N}$, ya que la mayor acumulación en este tejido ocurre antes de los 59 dds, sin embargo la absorción porcentual total del elemento difiere del $\mathrm{N}$, ya que la planta sigue el proceso de absorción de cantidades importantes de $\mathrm{P}$ (más del 50\%) en las etapas posteriores a los 59 dds. Este consumo tardío en la absorción de $\mathrm{P}$ indica que el follaje no tiene la capacidad de suministrar la totalidad del elemento que se requiere para la formación de la mazorca, por lo que continúa con la absorción en forma directa del suelo. Este nutrimento $(\mathrm{P})$ es necesario para la formación de sustancias como enzimas, ácidos nucleicos, etc., los cuales son requeridos en todas las etapas del ciclo de desarrollo de la planta y también en la etapa de formación del grano es necesario para la acumulación de fitina en la semilla, la cual es necesaria para alcanzar buen tamaño, número y viabilidad de las semillas (IPNI 1999). A nivel radical se presenta también un importante acúmulo en estas etapas posteriores a los 59 dds.

Por su parte el comportamiento del $\mathrm{K}$ se parece más al del $\mathrm{N}$, es decir la mayor absorción (cerca del 75\%) se da en las etapas anteriores a los 59 dds, y luego de esto se da un traslado del nutrimento desde el follaje hacia la formación de la mazorca; no obstante si se observan solamente los contenidos de $\mathrm{K}$ a nivel radical, este órgano acumula importantes cantidades porcentuales en etapas posteriores al inicio de formación de mazorca (cerca de un $40 \%$ del total absorbido por la raíz, dato no mostrado). 

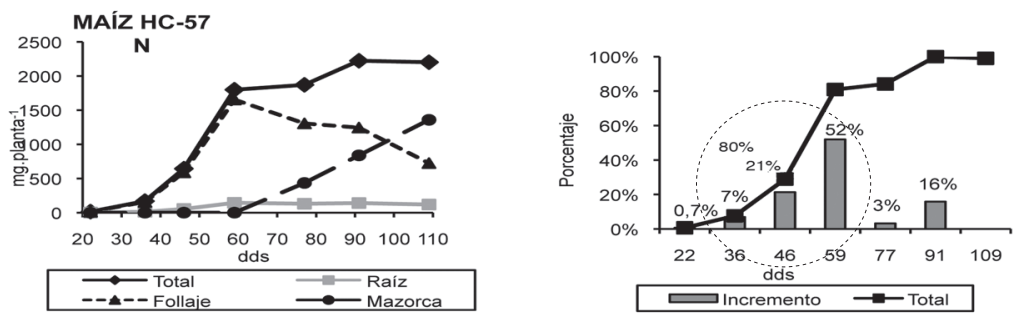

MAíz HC-57
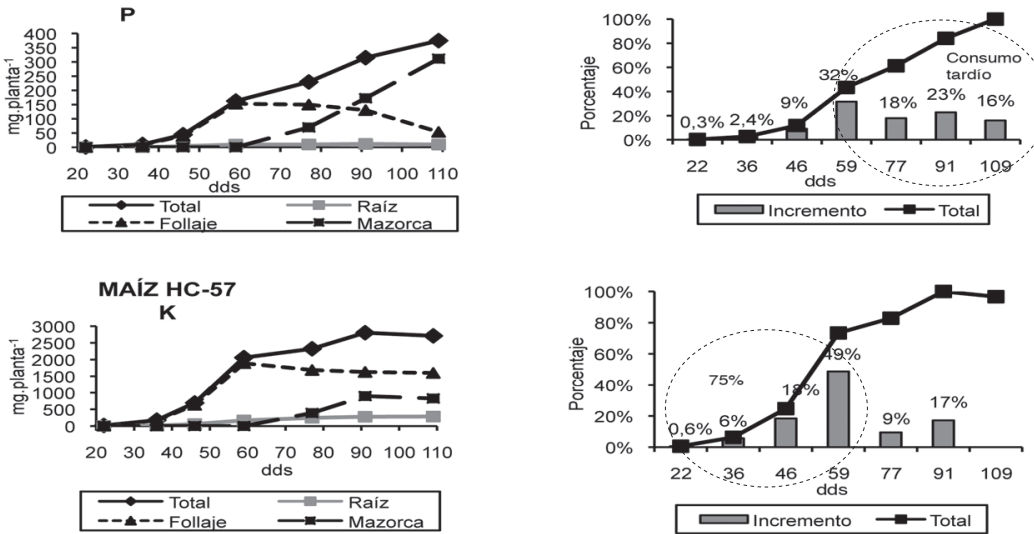

MAíz HC-57
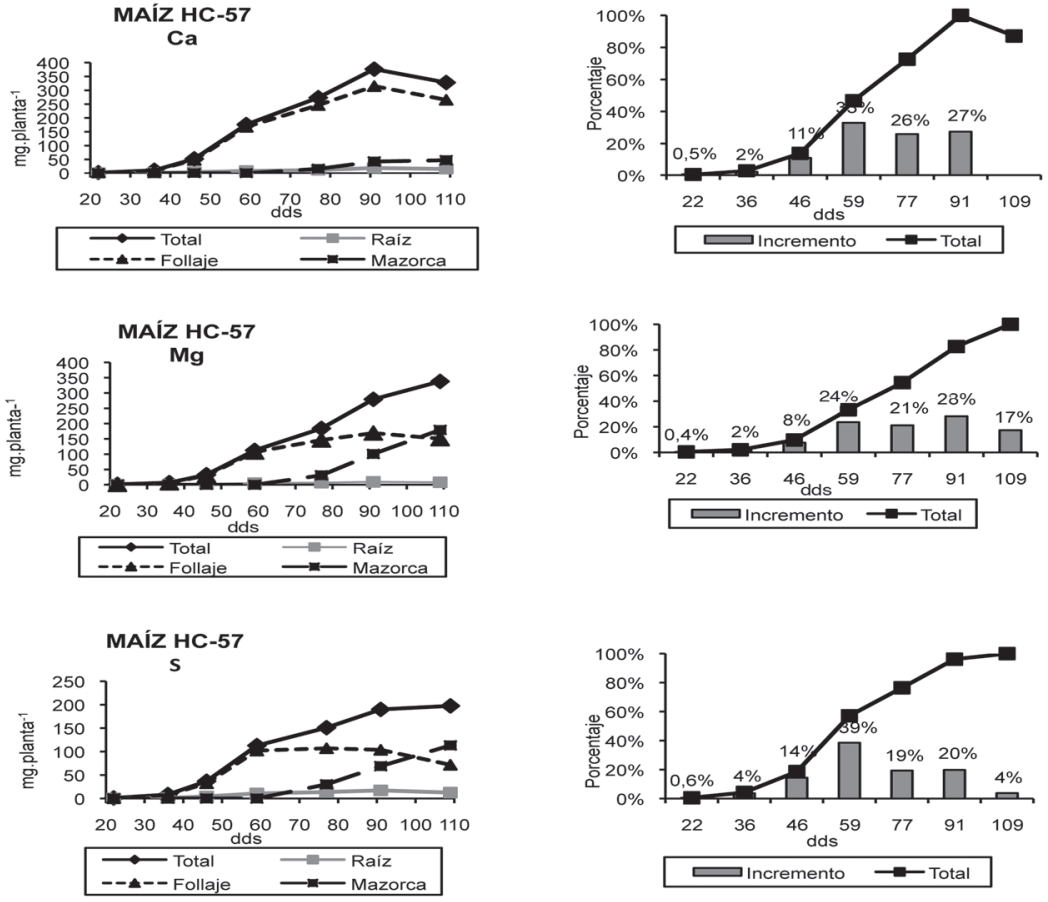

Fig. 3. Absorción total e incremento porcentual de macronutrimentos para las diferentes partes de la planta del híbrido de maíz HC-57 en la Estación Experimental Fabio Baudrit Moreno, Alajuela, Costa Rica. Año 2009. 

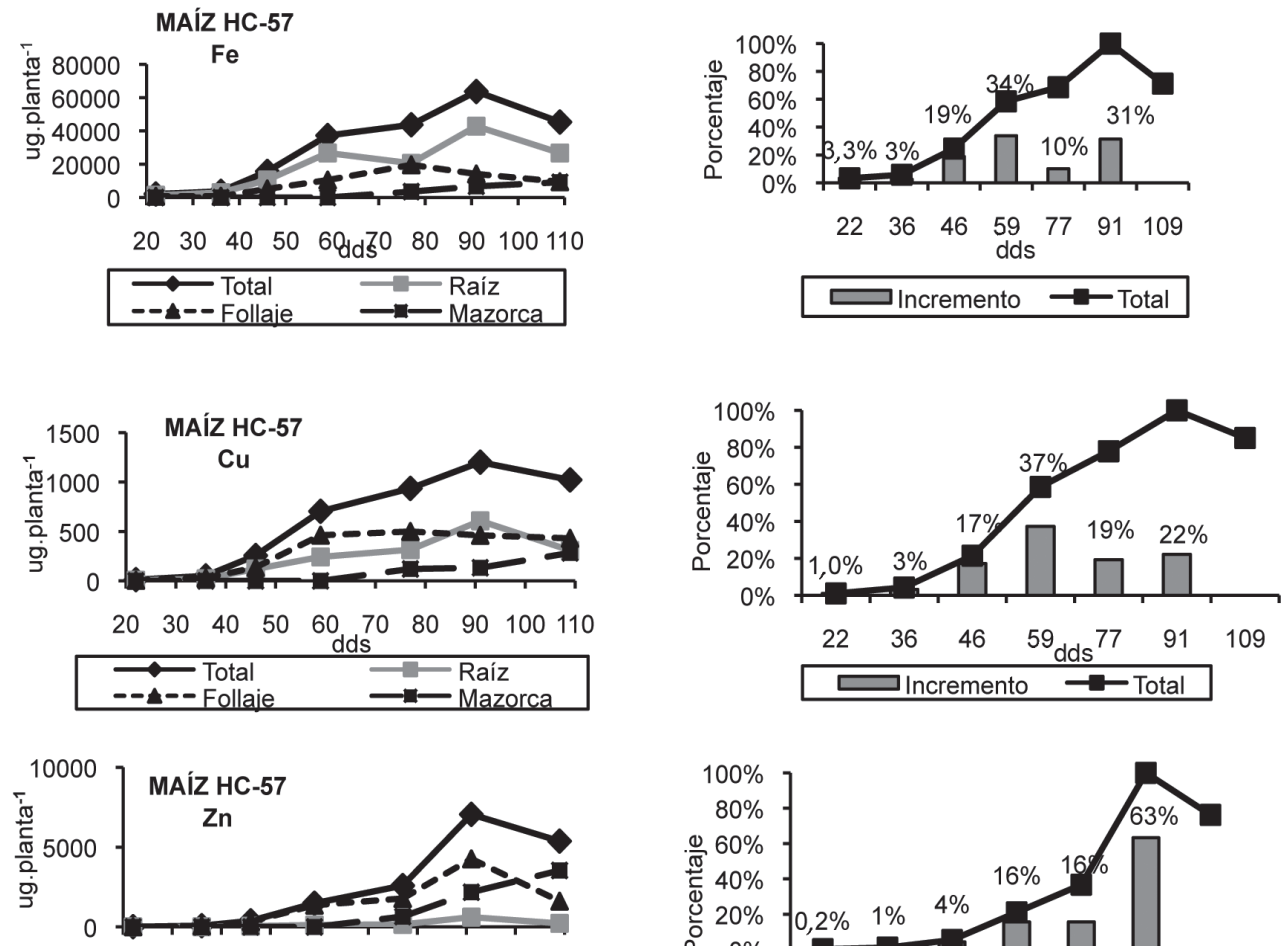

$\begin{array}{llllllllll}20 & 30 & 40 & 50 & 60 & 70 & 80 & 90 & 100 & 110\end{array}$

\begin{tabular}{|c|c|}
\hline Total & - Raíz \\
\hline
\end{tabular}
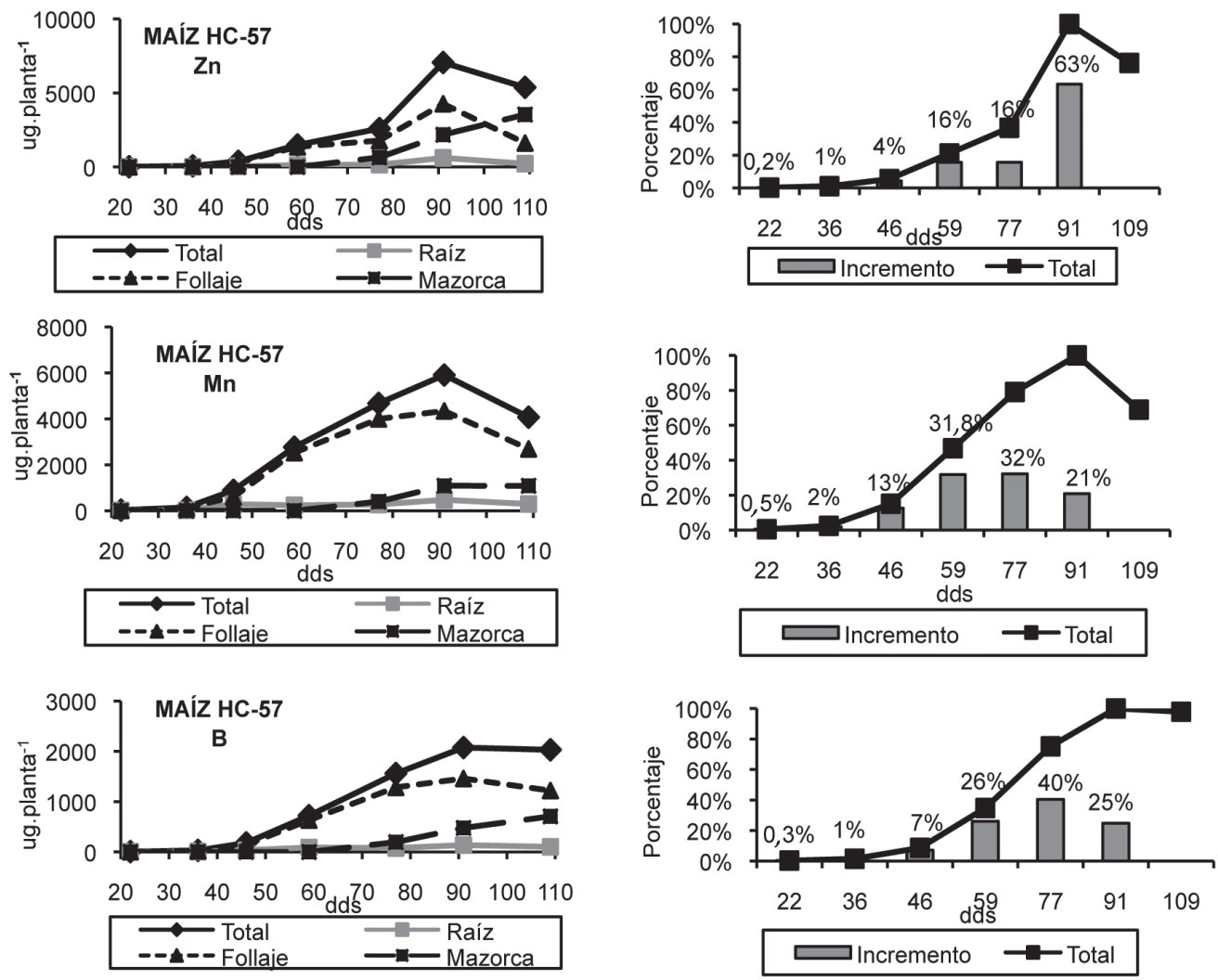

Fig. 4. Absorción total e incremento porcentual de micronutrimentos para las diferentes partes de la planta del híbrido de maíz HC-57 en la Estación Experimental Fabio Baudrit Moreno, Alajuela, Costa Rica. Año 2009. 
La absorción de $\mathrm{Ca}$ y $\mathrm{Mg}$ parece no depender de una etapa fenológica, ya que estos elementos se absorben de manera constante aún cuando se da la formación de la mazorca y su contenido en el follaje solo disminuyó en la última etapa evaluada, probablemente por un efecto de senescencia del tejido. En el caso del Ca este comportamiento es congruente con la función estructural de este elemento, ya que es constituyente esencial de los poligalacturonatos de $\mathrm{Ca}$ (Dordas 2008), los cuales dan estabilidad a las paredes celulares del tejido formado y del que se encuentra en formación.

El patrón mostrado por el $\mathrm{S}$ es similar al del $\mathrm{N}$ y K, no obstante, los requerimientos de $\mathrm{S}$ en las etapas posteriores a los 59 dds superan el $40 \%$ del total requerido por la planta durante su ciclo de desarrollo.

Es importante destacar que la absorción de todos estos elementos en las etapas anteriores a los 36 dds son en términos generales menores al $10 \%$ del total y en el caso de la mazorca su absorción se caracteriza por incrementos prácticamente lineales desde su formación hasta el final del ciclo. Solo en el caso del K se encontró una estabilización en su contenido en la etapa que abarca de R3 a madurez fisiológica.

\section{Micronutrimentos}

El comportamiento del HC-57 en la absorción total de microelementos, es como en la mayoría de los cultivos, más errática que la de los mayores, y no parece ser dependiente de los cambios reproductivos de la planta en algunos de los elementos, más bien, la planta tiende a acumular estos elementos en todas las etapas hasta los 91 dds (R3, 1254 GDT) y solamente en la etapa final se presenta una reducción en el contenido de todos los micronutrimentos (Figura 4).

La etapa de mayor necesidad de Mn y B en el follaje es la comprendida entre 46 y 59 dds (V9-VT, 632-811 GDT), que coincide con la etapa de polinización. Esto resulta de particular importancia, ya que según Brown y Hu (1998) la planta de maíz es una de las especies en las que el B no tiene movilidad, por lo tanto una aplicación foliar de $\mathrm{B}$ en estas etapas de mayor requerimiento podría contribuir a mejorar la producción de este cultivo. Además es conocido que este elemento tiene gran importancia en el proceso de polinización y un aumento en el rendimiento del cultivo del maíz (Ziaeyan y Rajaie 2009).

A nivel radical, entre los 77 y 91 dds, el híbrido incrementó su absorción de Mn y B en valores cercanos al $40 \%$ del máximo absorbido por este tejido.

La mayor demanda de Zn se presentó en las etapas finales del ciclo productivo del híbrido, lo cual tiene importancia no sólo desde el aspecto agronómico, sino también desde el punto de vista de nutrición de la población, pues a nivel mundial es reconocida la gran problemática de deficiencia de este elemento (Çakmak 2009). Un suplemento de $\mathrm{Zn}$ en las fases finales del cultivo beneficiaría tanto al cultivo como a la población que consumirá el producto.

\section{Análisis de translocación de nutrimentos $\mathbf{N}, \mathbf{P}$ y K}

Como se mencionó anteriormente, a los 59 dds ocurren 2 fenómenos relevantes: se produce el máximo desarrollo del follaje y a su vez, se inicia la formación de la mazorca. Al analizar los datos de absorción en valores absolutos alrededor de este momento, se encontró que existe relación entre la disminución del contenido de algunos nutrimentos en el follaje y el incremento de los mismos en la mazorca.

Para el caso del N, como se puede observar en la Figura 5, una gran cantidad de los requerimientos para la formación de la mazorca los suple el follaje. De los $435 \mathrm{mg}$ de $\mathrm{N}$ requeridos por la mazorca a los $77 \mathrm{dds}$, aproximadamente 349 son aportados por el follaje, lo cual representa la mayoría del requerimiento. En la etapa de los $91 \mathrm{dds}$ solo una pequeña cantidad $(61 \mathrm{mg})$ de lo requerido por la mazorca $(404 \mathrm{mg}$ ) es aportado por el follaje. En la etapa final las cantidades requeridas por la mazorca fueron aportadas completamente por el follaje.

Esta situación no se presentó con el $\mathrm{P}$, el cual en las etapas de los 77 y 91 dds debió ser 


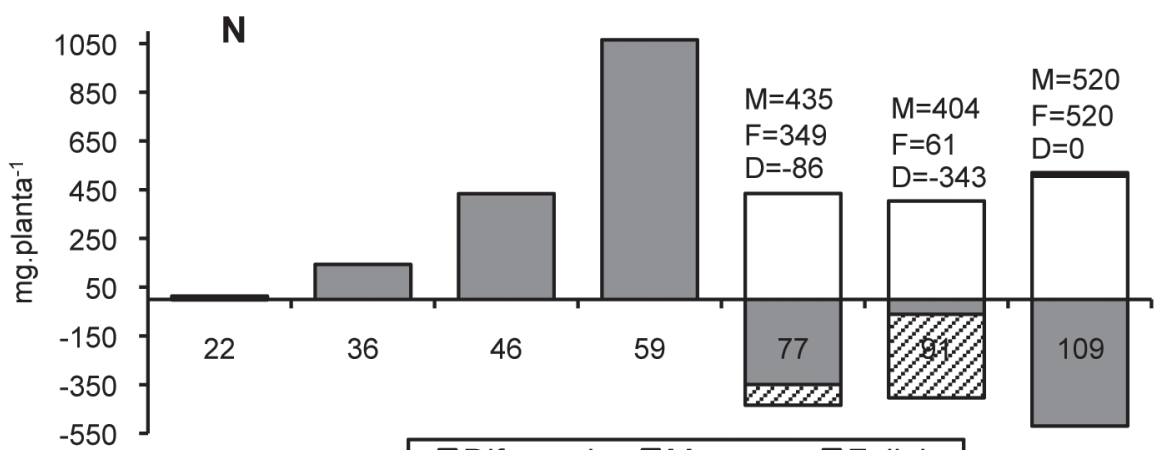

QDiferencia पMazorca QFollaie
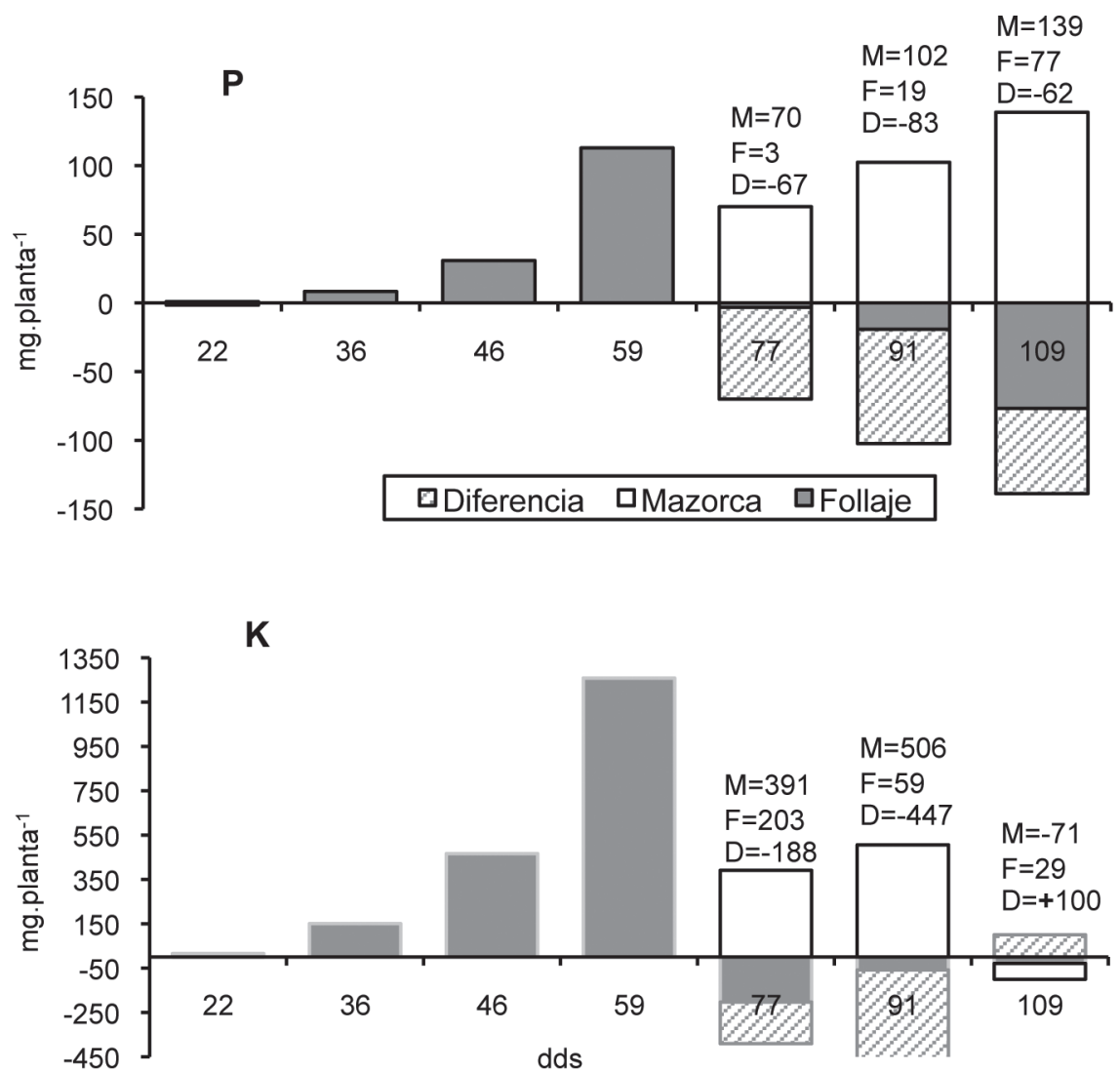

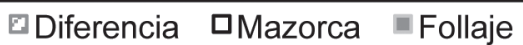

$\mathrm{M}=$ Aumento del nutrimento en mg en la mazorca. $\mathrm{F}=$ Pérdida del nutrimento en mg en el follaje (tallo y mazorca). $\mathrm{D}=$ Diferencia entre lo absorbido por la mazorca y lo perdido por el follaje.

Fig. 5. Relación entre la disminución del contenido de nutrimentos en el follaje y su respectivo incremento en la mazorca en el híbrido de maíz HC-57, Estación Experimental Fabio Baudrit Moreno, Alajuela, Costa Rica. 
aportado posiblemente por la absorción desde el suelo, ya que el follaje solo aportó un 3 y un $19 \%$ en cada etapa respectivamente, solamente en la etapa final, la de los 109 dds, se presentó un suministro bastante importante de $\mathrm{P}$ por el follaje, ya que aportó 77 de los $139 \mathrm{mg}$ requeridos por la mazorca en esta etapa.

Para el caso del K en la etapa de los 77 dds el follaje suple más del $50 \%$ de los requerimientos de la mazorca, sin embargo en la etapa posterior (91 dds) solo 59 de los $506 \mathrm{mg}$ requeridos por la mazorca fueron aportados por el follaje. En la última etapa tanto el follaje como la mazorca presentaron una disminución en el contenido de este elemento.

\section{Absorción total}

En el Cuadro 3 se resume para todos los elementos, la cantidad de nutrimentos absorbidos por la raíz, el follaje (tallo y hojas) y la mazorca en las diferentes etapas.

En el Cuadro 4 se resume la cantidad por hectárea absorbida por una plantación del híbrido HC-57 con un rendimiento de 50000 mazorcas.ha $^{-1}$; las cantidades se expresan en términos totales y por cosecha.

En el experimento se cosechó la mazorca, por lo que en forma directa no se obtuvo el rendimiento en grano, sin embargo si se asume que el $85 \%$ de esa mazorca llegara a ser grano cosechable al $14 \%$ de humedad y si se considera un $10 \%$ de pérdida, el rendimiento estimado para las 50000 mazorcas sería de aproximadamente 6,25 t.ha ${ }^{-1}$ de grano (14\% de humedad).

La utilidad de conocer estos requisitos del cultivo, radica en que se puede reponer al suelo la cantidad de nutrimentos que son extraídos mediante la cosecha de la mazorca, y evitaría la degradación química del suelo y además asegura la sostenibilidad del sistema productivo. Sin embargo esta información, no se debe convertir en el criterio único de fertilización, ya que en un sistema productivo intervienen muchos otros factores de tipo ambiental, físico, o hasta mineralógicos, los cuales podrían modificar la eficiencia de la fertilización.

En síntesis, las aplicaciones de $\mathrm{N}$ y $\mathrm{K}$ deben realizarse en etapas iniciales del ciclo de desarrollo del cultivo, ya que después de los 59 dds (V12-VT, 811 GDT) la demanda o absorción de nutrimentos es menor. Aplicaciones tardías de estos elementos, podrían no tener un efecto importante sobre el rendimiento del cultivo, sin embargo debe verificarse mediante ensayos de campo.

Es posible que este híbrido presente una buena respuesta a las aplicaciones de $\mathrm{P}$ en etapas intermedias y tardías del ciclo de desarrollo, ya que se presentó una importante absorción de este elemento en ambas etapas; esto también requiere de la evaluación experimental a nivel de campo antes de llevarse a nivel comercial.

Realizar aplicaciones foliares de B en las etapas cercanas a los 59 dds podría beneficiar al cultivo con una mejor polinización y aplicaciones foliares de $\mathrm{Zn}$ al final del ciclo ayudarían a mejorar el valor nutricional del producto cosechado.

El asocio de las curvas de absorción de nutrimentos a la información meteorológica a través del parámetro grados día temperatura resulta en una herramienta que puede colaborar en la extrapolación de la información generada en este estudio hacia otras zonas del país. 
Cuadro 3. Cantidad promedio de nutrimentos absorbidos en las etapas del ciclo de desarrollo del híbrido de maíz HC-57 y los respectivos grados día temperatura de cada etapa, Estación Experimental Fabio Baudrit Moreno, Alajuela, Costa Rica.

\begin{tabular}{|c|c|c|c|c|c|c|c|c|c|c|c|c|c|c|c|}
\hline \multirow{3}{*}{ Tejido } & \multirow{3}{*}{ dds } & \multirow{3}{*}{ GDT* } & \multirow{3}{*}{ Etapa fenológica } & \multirow{3}{*}{$\begin{array}{c}\text { Peso } \\
\text { seco } \\
(\mathrm{g})\end{array}$} & \multicolumn{11}{|c|}{ Cantidad Absorbida** } \\
\hline & & & & & \multicolumn{6}{|c|}{ mg.planta ${ }^{-1}$} & \multicolumn{5}{|c|}{$\mu \mathrm{g} \cdot$ planta $^{-1}$} \\
\hline & & & & & $\mathrm{N}$ & $\mathrm{P}$ & $\mathrm{K}$ & $\mathrm{Ca}$ & $\mathrm{Mg}$ & S & $\mathrm{Fe}$ & $\mathrm{Cu}$ & $\mathrm{Zn}$ & $\mathrm{Mn}$ & B \\
\hline \multirow{3}{*}{ Mazorca } & 77 & 1062 & R1-R2 & 22,6 & 435 & 70 & 391 & 16 & 31 & 30 & 3518 & 121 & 648 & 399 & 196 \\
\hline & 91 & 1254 & $\mathrm{R} 3$ & 79,6 & 839 & 172 & 897 & 42 & 101 & 69 & 6714 & 133 & 2176 & 1088 & 478 \\
\hline & 109 & 1501 & Madurez fisiológica & 141,5 & 1359 & 311 & 826 & 47 & 179 & 113 & 9152 & 283 & 3538 & 1085 & 708 \\
\hline \multirow{7}{*}{$\begin{array}{l}\text { Tallo y } \\
\text { hojas }\end{array}$} & 22 & 318 & V5 & 0,3 & 13 & 1 & 16 & 2 & 1 & 1 & 733 & 5 & 10 & 19 & 3 \\
\hline & 36 & 501 & V7 & 4,7 & 156 & 9 & 166 & 10 & 6 & 7 & 772 & 30 & 68 & 120 & 23 \\
\hline & 46 & 632 & V9 & 21,6 & 590 & 40 & 632 & 48 & 30 & 32 & 5145 & 137 & 331 & 604 & 144 \\
\hline & 59 & 811 & V12-VT & 72,9 & 1655 & 153 & 1891 & 168 & 107 & 102 & 10475 & 462 & 1361 & 2528 & 632 \\
\hline & 77 & 1062 & R1-R2 & 107,0 & 1306 & 150 & 1688 & 246 & 146 & 107 & 19623 & 499 & 1784 & 3996 & 1284 \\
\hline & 91 & 1254 & $\mathrm{R} 3$ & 115,2 & 1245 & 131 & 1629 & 315 & 169 & 104 & 14137 & 461 & 4264 & 4341 & 1460 \\
\hline & 109 & 1501 & Madurez fisiológica & 107,6 & 725 & 54 & 1600 & 265 & 151 & 72 & 9470 & 430 & 1614 & 2690 & 1220 \\
\hline \multirow{7}{*}{ Raíz } & 22 & 318 & V5 & 0,1 & 2 & 0,2 & 2 & 0,3 & 0,2 & 0,2 & 1352 & 7 & 4 & 12 & 4 \\
\hline & 36 & 501 & V7 & 0,6 & 12 & 1 & 10 & 1 & 1 & 1 & 2934 & 21 & 10 & 25 & 8 \\
\hline & 46 & 632 & V9 & 3,1 & 54 & 4 & 60 & 4 & 3 & 4 & 10537 & 120 & 50 & 286 & 36 \\
\hline & 59 & 811 & V12-VT & 9,2 & 144 & 10 & 168 & 8 & 6 & 11 & 26764 & 243 & 123 & 239 & 89 \\
\hline & 77 & 1062 & R1-R2 & 14,8 & 131 & 10 & 245 & 11 & 7 & 14 & 20562 & 316 & 158 & 276 & 79 \\
\hline & 91 & 1254 & $\mathrm{R} 3$ & 17,9 & 141 & 12 & 279 & 19 & 10 & 17 & 42844 & 607 & 613 & 476 & 137 \\
\hline & 109 & 1501 & Madurez fisiológica & 17,1 & 119 & 10 & 285 & 15 & 8 & 13 & 26739 & 308 & 223 & 297 & 103 \\
\hline \multirow{7}{*}{ Total } & 22 & 318 & V5 & 0,4 & 15 & 1 & 17 & 2 & 1 & 1 & 2084 & 12 & 14 & 31 & 7 \\
\hline & 36 & 501 & V7 & 5,3 & 169 & 10 & 176 & 11 & 7 & 8 & 3705 & 51 & 78 & 145 & 32 \\
\hline & 46 & 632 & V9 & 24,6 & 644 & 44 & 692 & 52 & 33 & 37 & 15682 & 257 & 381 & 890 & 180 \\
\hline & 59 & 811 & V12-VT & 82,1 & 1799 & 163 & 2058 & 176 & 112 & 113 & 37239 & 704 & 1484 & 2767 & 721 \\
\hline & 77 & 1062 & $\mathrm{R} 1-\mathrm{R} 2$ & 144,4 & 1871 & 230 & 2324 & 273 & 184 & 151 & 43703 & 936 & 2590 & 4671 & 1559 \\
\hline & 91 & 1254 & $\mathrm{R} 3$ & 212,7 & 2224 & 315 & 2804 & 377 & 279 & 190 & 63695 & 1201 & 7054 & 5905 & 2074 \\
\hline & 109 & 1501 & Madurez fisiológica & 266,3 & 2202 & 375 & 2710 & 328 & 338 & 198 & 45361 & 1022 & 5375 & 4072 & 2030 \\
\hline $\begin{array}{l}\text { Máximo } \\
\text { total }\end{array}$ & & 1501 & & 266,3 & 2224 & 375 & 2804 & 377 & 338 & 198 & 63695 & 1201 & 7054 & 5905 & 2074 \\
\hline
\end{tabular}

* Grados día se presenta el acumulado de las diferentes etapas.

** Cantidad absorbida contempla el total de cada nutrimento en cada etapa, no desestima absorción de etapas anteriores. dds: días después de la siembra.

GDT: Grados día temperatura con una temperatura base mínima de $10^{\circ} \mathrm{C}$ y máxima de $30^{\circ} \mathrm{C}$. 
Cuadro 4. Cantidad estimada del requerimiento de nutrimentos por la planta y por la cosecha del híbrido de maíz HC-57 con un rendimiento aproximado de 50000 mazorcas por hectárea, Estación Experimental Fabio Baudrit Moreno, Alajuela, Costa Rica.

\begin{tabular}{lccccccccccc}
\hline \multirow{2}{*}{ Sección } & & \multicolumn{1}{c}{ kg.ha ${ }^{-1}$} & & & & & \\
& $\mathrm{~N}$ & $\mathrm{P}$ & $\mathrm{K}$ & $\mathrm{Ca}$ & $\mathrm{Mg}$ & $\mathrm{S}$ & $\mathrm{Fe}$ & $\mathrm{Cu}$ & $\mathrm{Zn}$ & $\mathrm{Mn}$ & $\mathrm{B}$ \\
\hline Total & 111,2 & 18,7 & 140,2 & 18,8 & 16,9 & 9,9 & 3,2 & 0,1 & 0,4 & 0,3 & 0,1 \\
Mazorca & 67,9 & 15,6 & 44,8 & 2,4 & 9,0 & 5,7 & 0,46 & 0,01 & 0,18 & 0,05 & 0,04 \\
& $\mathrm{~N}$ & $\mathrm{P}_{2} \mathrm{O}_{5}$ & $\mathrm{~K}_{2} \mathrm{O}$ & $\mathrm{CaO}$ & $\mathrm{MgO}$ & $\mathrm{S}$ & $\mathrm{Fe}$ & $\mathrm{Cu}$ & $\mathrm{Zn}$ & $\mathrm{Mn}$ & $\mathrm{B}$ \\
\hline Total & 111,2 & 42,9 & 168,3 & 26,4 & 28,0 & 9,9 & 3,2 & 0,1 & 0,4 & 0,3 & 0,1 \\
Mazorca & 67,9 & 35,6 & 53,8 & 3,3 & 14,9 & 9,9 & 3,2 & 0,1 & 0,4 & 0,3 & 0,1 \\
\hline
\end{tabular}

El rendimiento de 50000 mazorcas.ha ${ }^{-1}$ se estima que puede equipararse a 6,25 t.ha ${ }^{-1}$ de grano al $14 \%$ de humedad.

\section{LITERATURA CITADA}

APS 1984. Compendium of corn diseases. The American Phytopathological Society. 2 ed. 105 p.

AVILÁN L., RODRÍGUEZ M. 1995. Época de floración y cosecha del aguacate en la región norte de Venezuela. Agronomía Tropical 45(1):35-50.

BARRIOS E., LÓPEZ C. 2009. Temperatura base y tasa de extensión foliar en frijol. Agrociencia 43(1):29-35.

BERTSCH F. 2003. Absorción de nutrimentos por los cultivos. San José, ACCS. 307 p.

BROWN P., HU HENNING. 1998. Boron mobility and consequent management in different crops. Better Crops 82(2):29-31.

ÇAKMAK I. 2009. Enrichment of fertilizers with zinc: An excellent investment for humanity and crop production in India. Journal of Trace Elements in Medicine and Biology 23(4):281-289.

CASASOLA S., TIESSEN A. 2009. Efectos de la influencia ambiental y genética en la fisiología y desarrollo en diferentes variedades de maíz (Zea mays). Memorias del Programa de Verano de la Ciencia, Universidad Autónoma de Querétaro, México.

CORRALES M., BERTSCH F., BEJARANO J. 2005. Los laboratorios de análisis de suelos y foliares en Costa Rica: Informe del comité de laboratorios de análisis de suelos, plantas y aguas. Agronomía Costarricense 29(3):125-135.
DORDAS C. 2008. Role of nutrients in controlling plant diseases in sustainable agriculture. A review. Agron. Sustain. Dev. 28:33-46.

GARCÍA F. 2005. Criterios para el manejo de la fertilización del cultivo de maíz. IPNI. 19 p.

HAMADA E., SILVEIRA PINTO H. 2001. Avaliação do desenvolvimento do trigo utilizando medidas radiométricas em função de graus-dia. Anais $\mathrm{X}$ SBSR, Foz de Iguazú, INPE. p 95-101.

HOLDRIDGE L. 1982. Ecología basada en zonas de vida. IICA, San José Costa Rica. 216 p.

IOWA STATE UNIVERSITY. 1992. How a corn plant develops. Iowa State University of Science and Technology, Cooperative Extension Service. Special report $\mathrm{N}^{\circ} .48$.

IPNI. 1999. Functions of phosphorus in plants. Better Crops 83(1):6-7.

MILLER P., BRANDT S. 2001. Using growing degree days to predict plant stages. Montguide Extension Service, Montana State University. 8 p.

MISLE E. 2003. Caracterización termofisiológica del ritmo de absorción de nutrientes del melón (Cucumis melo L., var reticulatus Naud). Cien. Inv. Agr. 30(1):39-50.

REVISTA GIRASOL. 2006. Semillas del futuro. Revista Girasol 9(31):3-6.

RODRÍGUEZ W., FLÓREZ V. 2006. Comportamiento fenológico de tres variedades de rosas rojas en 
función de la acumulación de la temperatura. Agronomía Colombiana 24(2):247-257.

WIEBOLD B. sa. Growing degree days and corn maturity. Corn Facts, MU Plant Sciences Extension Web Site, College of Agriculture, Food and Natural Resources, University of Missouri. http://www.psu.missouri. edu/cornx.
YZARRA W., TREBEJO I., NORIEGA V. 2009. Evaluación de unidades térmicas para el crecimiento y desarrollo del cultivo de maíz amarillo duro (Zea mays L.) en la costa central del Perú. Revista Peruana GeoAtmosférica RPGA (1):1-10.

ZIAEYAN A., RAJAIE M. 2009. Combined effect of Zinc and Boron on yield and nutrients accumulation in corn. International Journal of Plant Production 3(3):35-44. 
